\title{
Computational Electrochemistry of Ruthenium Anticancer Agents. Unprecedented Benchmarking of Implicit Solvation Methods
}

\section{Supporting Information}

Table S-1. Mean unsigned errors (MUE) in the calculation of SRPs using protocols I-VII. All values in $\mathrm{kcal} / \mathrm{mol}$.

\begin{tabular}{|c|c|c|c|c|c|c|c|c|}
\hline compounds & number & $\begin{array}{c}\text { I } \\
\text { PCM } \\
\text { UA0 }\end{array}$ & $\begin{array}{c}\text { II } \\
\text { PCM } \\
\text { UAHF }\end{array}$ & $\begin{array}{c}\text { III } \\
\text { PCM } \\
\text { UAKS }\end{array}$ & $\begin{array}{c}\text { IV } \\
\text { PCM } \\
\text { Bondi } \\
\end{array}$ & $\begin{array}{c}\mathrm{V} \\
\mathrm{CPCM} \\
\mathrm{UAKS}\end{array}$ & $\begin{array}{c}\text { VI } \\
\text { CPCM } \\
\text { Bondi }\end{array}$ & $\begin{array}{l}\text { VII } \\
\text { PBF }\end{array}$ \\
\hline $\begin{array}{c}\text { all } \\
\text { solvent }\end{array}$ & 80 & 5.4 & 7.4 & 7.1 & 4.3 & 7.0 & 4.4 & 3.7 \\
\hline $\mathrm{ACN}$ & 4 & 2.3 & 14.8 & 14.9 & 0.6 & 14.8 & 0.7 & 0.5 \\
\hline DMF & 16 & 2.3 & 3.7 & 3.6 & 5.7 & 3.5 & 6.0 & 3.6 \\
\hline DMSO & 14 & 7.8 & 8.7 & 8.2 & 4.8 & 8.1 & 5.0 & 3.9 \\
\hline $\begin{array}{l}\text { water } \\
\text { charge }\end{array}$ & 46 & 6.0 & 7.6 & 7.2 & 4.0 & 7.1 & 4.0 & 4.0 \\
\hline$+3 /+2$ & 8 & 10.4 & 7.0 & 6.2 & 6.2 & 6.1 & 6.1 & 5.3 \\
\hline$+2 /+1$ & 18 & 4.3 & 4.0 & 3.7 & 3.4 & 3.6 & 3.4 & 3.1 \\
\hline$+1 / 0$ & 10 & 2.4 & 3.4 & 3.0 & 4.3 & 3.0 & 4.3 & 4.3 \\
\hline $0 /-1$ & 21 & 6.0 & 5.6 & 5.4 & 4.2 & 5.3 & 4.4 & 2.5 \\
\hline$-1 /-2$ & 23 & 5.2 & 13.5 & 13.2 & 4.6 & 13.0 & 4.6 & 4.4 \\
\hline Lead $^{\mathrm{a}}$ & 14 & 6.8 & 14.6 & 14.0 & 3.3 & 13.9 & 3.1 & 3.7 \\
\hline
\end{tabular}


Table S-2. Mean signed errors (MSE) in the calculation of SRPs using protocols I-VII. All values in $\mathrm{kcal} / \mathrm{mol}$.

\begin{tabular}{ccccrrrrr} 
& & \multicolumn{1}{c}{ I } & II & III & IV & V & VI & VII \\
compounds & number & UA0 & PCM & PCM & PCM & CPCM & CPCM & PBF \\
\hline all & 80 & 0.2 & -3.8 & -3.9 & 1.7 & -3.8 & 1.9 & 0.7 \\
solvent & & & & & & & & \\
$A C N$ & 4 & -2.3 & -14.8 & -14.9 & -0.2 & -14.8 & 0.1 & 0.1 \\
$D M F$ & 16 & 1.3 & -2.6 & -2.5 & 5.3 & -2.4 & 5.6 & 2.6 \\
UMSO & 14 & -4.1 & -7.7 & -7.7 & 4.8 & -7.5 & 5.0 & 3.4 \\
water & 46 & 1.3 & -2.2 & -2.2 & -0.3 & -2.2 & -0.2 & -0.8 \\
charge & & & & & & & & \\
$+3 /+2$ & 8 & 10.4 & 6.7 & 5.8 & 4.6 & 5.7 & 4.5 & 4.7 \\
$+2 /+1$ & 18 & 3.2 & 3.2 & 2.7 & -1.6 & 2.6 & -1.6 & -1.1 \\
$+1 / 0$ & 10 & 0.8 & -0.1 & -0.5 & 2.3 & -0.5 & 2.4 & -0.4 \\
0/-1 & 21 & -2.7 & -5.1 & -4.6 & 4.0 & -4.5 & 4.3 & 2.2 \\
$-1 /-2$ & 23 & -3.5 & -13.5 & -13.2 & 1.0 & -13.0 & 1.2 & -0.1 \\
& & & & & & & & \\
Lead $^{\text {a }}$ & 14 & -6.8 & -14.6 & -14.0 & -2.7 & -13.9 & -2.6 & -3.7 \\
\hline
\end{tabular}

${ }^{a}$ Aqueous SRPs of the compounds with the lead structure trans-[ $\left.\mathrm{RuCl}_{4}(\mathrm{~L})\left(\mathrm{L}^{\prime}\right)\right]^{-}$

Table S-3. Calculated vs experimental SRPs. Protocol I: PCM/UA0; II: PCM/UAHF; III: PCM/UAKS; IV: PCM/Bondi; V: CPCM/UAKS; VI: CPCM/Bondi; VII: PBF. The results are sorted by molecular charge, solvent, and chemical similarity.

\begin{tabular}{|c|c|c|c|c|c|c|c|c|c|c|}
\hline & & & & & & & VI & & & \\
\hline & & PCM & PCM & PCM & PCM & $\mathrm{CPCM}$ & $\mathrm{CPCM}$ & PBF & & \\
\hline Complex $^{a}$ (oxidized form) & solvent & UA0 & UAHF & UAKS & Bondi & UAKS & Bondi & PBF & $\exp$ & Ref \\
\hline$\left[\operatorname{Ru}(\operatorname{Im})_{6}\right]^{3+}$ & water & 0.51 & 0.66 & 0.56 & 0.24 & 0.56 & 0.24 & 0.42 & 0.30 & $24 \mathrm{~g}$ \\
\hline$\left[\mathrm{Ru}(\mathrm{MeIm})_{6}\right]^{3+}$ & water & 0.40 & 0.46 & 0.36 & 0.06 & 0.36 & 0.06 & 0.16 & 0.28 & $24 \mathrm{~g}$ \\
\hline$\left[\operatorname{Ru}(\mathrm{en})_{3}\right]^{3+}$ & water & 0.46 & 0.29 & 0.27 & 0.23 & 0.27 & 0.23 & 0.23 & 0.18 & $24 \mathrm{~b}$ \\
\hline$\left[\mathrm{Ru}\left(\mathrm{OH}_{2}\right)_{6}\right]^{3+}$ & water & 1.16 & 0.16 & 0.16 & 1.14 & 0.15 & 1.14 & 1.06 & 0.23 & 59 \\
\hline$\left[\mathrm{Ru}\left(\mathrm{NH}_{3}\right)_{5}\left(\mathrm{OH}_{2}\right)\right]^{3+}$ & water & 0.68 & 0.53 & 0.53 & 0.34 & 0.53 & 0.34 & 0.25 & 0.08 & $24 \mathrm{~b}$ \\
\hline$c i s-\left[\mathrm{Ru}\left(\mathrm{NH}_{3}\right)_{4}(\mathrm{Im})_{2}\right]^{3+}$ & water & 0.52 & 0.57 & 0.56 & 0.23 & 0.56 & 0.22 & 0.27 & 0.15 & $24 \mathrm{~g}$ \\
\hline trans $-\left[\mathrm{Ru}\left(\mathrm{NH}_{3}\right)_{4}(\mathrm{Im})_{2}\right]^{3+}$ & water & 0.53 & 0.59 & 0.54 & 0.27 & 0.54 & 0.27 & 0.26 & 0.12 & $24 \mathrm{~g}$ \\
\hline$c i s-\left[\mathrm{Ru}\left(\mathrm{NH}_{3}\right)_{4}\left(\mathrm{OH}_{2}\right)_{2}\right]^{3+}$ & water & 0.79 & 0.48 & 0.47 & 0.51 & 0.46 & 0.51 & 0.40 & 0.11 & $24 \mathrm{~b}$ \\
\hline$\left[\mathrm{RuCl}\left(\mathrm{OH}_{2}\right)_{5}\right]^{2+}$ & water & 0.56 & 0.66 & 0.62 & 0.61 & 0.62 & 0.61 & 0.52 & 0.08 & $24 \mathrm{~b}$ \\
\hline$\left[\mathrm{Ru}\left(\mathrm{NH}_{3}\right)_{5} \mathrm{Br}\right]^{2+}$ & water & 0.16 & 0.13 & 0.13 & -0.07 & 0.13 & -0.07 & -0.11 & -0.02 & $24 \mathrm{a}$ \\
\hline$\left[\mathrm{Ru}\left(\mathrm{NH}_{3}\right)_{5} \mathrm{Cl}\right]^{2+}$ & water & 0.08 & 0.01 & 0.01 & -0.15 & 0.01 & -0.15 & -0.20 & -0.06 & $24 b$ \\
\hline$\left[\mathrm{Ru}\left(\mathrm{NH}_{3}\right)_{5}(\mathrm{OH})\right]^{2+}$ & water & -0.75 & -0.75 & -0.75 & -0.86 & -0.75 & -0.86 & -0.93 & -0.41 & $24 \mathrm{~b}$ \\
\hline$c i s-\left[\mathrm{Ru}\left(\mathrm{NH}_{3}\right)_{4} \mathrm{Cl}(\mathrm{py})\right]^{2+}$ & water & 0.28 & 0.25 & 0.25 & 0.06 & 0.25 & 0.06 & 0.09 & 0.17 & $24 \mathrm{a}$ \\
\hline trans $-\left[\mathrm{Ru}\left(\mathrm{NH}_{3}\right)_{4} \mathrm{Cl}(\mathrm{py})\right]^{2+}$ & water & 0.28 & 0.26 & 0.26 & 0.08 & 0.26 & 0.08 & -0.05 & 0.20 & $24 \mathrm{a}$ \\
\hline$c i s-\left[\mathrm{Ru}\left(\mathrm{NH}_{3}\right)_{4} \mathrm{Cl}(\mathrm{isn})\right]^{2+}$ & water & 0.47 & 0.41 & 0.45 & 0.26 & 0.42 & 0.26 & 0.25 & 0.23 & $24 \mathrm{a}$ \\
\hline
\end{tabular}




\begin{tabular}{|c|c|c|c|c|c|c|c|c|c|c|}
\hline trans- $\left[\mathrm{Ru}\left(\mathrm{NH}_{3}\right)_{4} \mathrm{Cl}(\mathrm{isn})\right]^{2+}$ & water & 0.36 & 0.36 & 0.34 & 0.17 & 0.34 & 0.17 & 0.14 & 0.29 & $24 \mathrm{a}$ \\
\hline$c i s-\left[\mathrm{Ru}\left(\mathrm{NH}_{3}\right)_{4}\left(\mathrm{CH}_{3} \mathrm{CN}\right) \mathrm{Cl}\right]^{2+}$ & water & 0.45 & 0.38 & 0.36 & 0.21 & 0.36 & 0.21 & 0.21 & 0.31 & $24 \mathrm{a}$ \\
\hline trans $-\left[\mathrm{Ru}\left(\mathrm{NH}_{3}\right)_{4}\left(\mathrm{CH}_{3} \mathrm{CN}\right) \mathrm{Cl}\right]^{2+}$ & water & 0.52 & 0.47 & 0.47 & 0.28 & 0.46 & 0.28 & 0.23 & 0.32 & $24 \mathrm{a}$ \\
\hline$c i s-\left[\mathrm{Ru}\left(\mathrm{NH}_{3}\right)_{4} \mathrm{Br}(\mathrm{py})\right]^{2+}$ & water & 0.36 & 0.32 & 0.32 & 0.11 & 0.32 & 0.11 & 0.14 & 0.19 & $24 \mathrm{a}$ \\
\hline trans $-\left[\mathrm{Ru}\left(\mathrm{NH}_{3}\right)_{4} \mathrm{Br}(\mathrm{py})\right]^{2+}$ & water & 0.33 & 0.32 & 0.32 & 0.12 & 0.32 & 0.12 & 0.11 & 0.19 & $24 \mathrm{a}$ \\
\hline cis- $\left[\mathrm{Ru}\left(\mathrm{NH}_{3}\right)_{4} \mathrm{Br}(\mathrm{isn})\right]^{2+}$ & water & 0.47 & 0.41 & 0.46 & 0.29 & 0.46 & 0.29 & 0.27 & 0.24 & $24 \mathrm{a}$ \\
\hline trans $-\left[\mathrm{Ru}\left(\mathrm{NH}_{3}\right)_{4} \mathrm{Br}(\mathrm{isn})\right]^{2+}$ & water & 0.33 & 0.39 & 0.32 & 0.09 & 0.32 & 0.09 & 0.19 & 0.30 & $24 \mathrm{a}$ \\
\hline$c i s-\left[\mathrm{Ru}\left(\mathrm{NH}_{3}\right)_{4} \mathrm{I}(\mathrm{py})\right]^{2+}$ & water & 0.41 & 0.39 & 0.40 & 0.19 & 0.39 & 0.19 & 0.25 & 0.23 & $24 \mathrm{a}$ \\
\hline trans $-\left[\mathrm{Ru}\left(\mathrm{NH}_{3}\right)_{4} \mathrm{I}(\mathrm{py})\right]^{2+}$ & water & 0.10 & 0.45 & 0.13 & -0.25 & 0.13 & -0.26 & 0.26 & 0.18 & $24 \mathrm{a}$ \\
\hline$c i s-\left[\mathrm{Ru}\left(\mathrm{NH}_{3}\right)_{4} \mathrm{I}(\mathrm{isn})\right]^{2+}$ & water & 0.53 & 0.48 & 0.49 & 0.34 & 0.49 & 0.34 & 0.37 & 0.26 & $24 \mathrm{a}$ \\
\hline trans $-\left[\mathrm{Ru}\left(\mathrm{NH}_{3}\right)_{4} \mathrm{I}(\mathrm{isn})\right]^{2+}$ & water & 0.55 & 0.54 & 0.53 & 0.30 & 0.52 & 0.30 & 0.35 & 0.29 & $24 \mathrm{a}$ \\
\hline cis- $\left[\mathrm{Ru}\left(\mathrm{NH}_{3}\right)_{4} \mathrm{Cl}_{2}\right]^{+}$ & water & -0.25 & -0.41 & -0.41 & -0.31 & -0.41 & -0.30 & -0.71 & -0.11 & $24 \mathrm{~b}$ \\
\hline trans $-\left[\mathrm{Ru}\left(\mathrm{NH}_{3}\right)_{4} \mathrm{Cl}_{2}\right]^{+}$ & water & -0.14 & -0.34 & -0.34 & -0.24 & -0.34 & -0.23 & -0.23 & -0.16 & $24 \mathrm{a}$ \\
\hline cis- $\left[\mathrm{RuCl}_{2}\left(\mathrm{OH}_{2}\right)_{4}\right]^{+}$ & water & 0.05 & 0.06 & 0.06 & 0.27 & 0.06 & 0.27 & 0.25 & 0.03 & $24 \mathrm{~b}$ \\
\hline trans $-\left[\mathrm{RuCl}_{2}(\mathrm{Im})_{4}\right]^{+}$ & $D M F$ & -0.20 & -0.17 & -0.19 & 0.13 & -0.19 & 0.14 & -0.24 & -0.15 & $25 \mathrm{c}$ \\
\hline trans $-\left[\mathrm{Ru}(\mathrm{bim})_{4} \mathrm{Cl}_{2}\right]^{+}$ & $D M F$ & 0.28 & 0.28 & 0.24 & 0.43 & 0.25 & 0.44 & 0.25 & -0.03 & $25 \mathrm{c}$ \\
\hline trans $-\left[\mathrm{RuCl}_{2}(\operatorname{trz})_{4}\right]^{+}$ & $D M F$ & 0.31 & 0.30 & 0.31 & 0.42 & 0.31 & 0.42 & 0.18 & 0.24 & $25 \mathrm{c}$ \\
\hline trans $-\left[\mathrm{RuCl}_{2}(\mathrm{pz})_{4}\right]^{+}$ & $D M F$ & 0.23 & 0.18 & 0.21 & 0.22 & 0.21 & 0.22 & 0.22 & 0.35 & $25 \mathrm{c}$ \\
\hline trans $-\left[\mathrm{RuCl}_{2}(\mathrm{Im})_{4}\right]^{+}$ & DMSO & -0.23 & -0.27 & -0.29 & -0.17 & -0.29 & -0.17 & -0.24 & -0.18 & $25 \mathrm{c}$ \\
\hline trans $-\left[\mathrm{Ru}(\mathrm{bim})_{4} \mathrm{Cl}_{2}\right]^{+}$ & $D M S O$ & 0.20 & 0.19 & 0.10 & 0.23 & 0.10 & 0.24 & 0.31 & -0.03 & $25 \mathrm{c}$ \\
\hline trans $-\left[\mathrm{RuCl}_{2}(\operatorname{trz})_{4}\right]^{+}$ & DMSO & 0.22 & 0.28 & 0.23 & 0.18 & 0.23 & 0.18 & 0.17 & 0.20 & $25 \mathrm{c}$ \\
\hline $\mathrm{Ru}(\mathrm{bpy})\left(4,4^{\prime}-\mathrm{bpy}\right) \mathrm{Cl}_{3}$ & $A C N$ & 0.06 & -0.36 & -0.33 & 0.12 & -0.32 & 0.13 & 0.14 & 0.18 & $24 \mathrm{i}$ \\
\hline $\mathrm{Ru}(\mathrm{bpy})\left(\mathrm{CH}_{3} \mathrm{CN}\right) \mathrm{Cl}_{3}$ & $A C N$ & 0.14 & -0.42 & -0.42 & 0.23 & -0.41 & 0.24 & 0.23 & 0.21 & $24 \mathrm{i}$ \\
\hline mer,trans $-\mathrm{RuCl}_{3}\left(\mathrm{Me}_{2} \mathrm{~S}\right)(\mathrm{Ind})_{2}$ & $D M F$ & 0.11 & 0.04 & 0.11 & 0.25 & 0.06 & 0.24 & 0.21 & 0.16 & $25 b$ \\
\hline mer, trans $-\mathrm{RuCl}_{3}\left(\mathrm{Et}_{2} \mathrm{~S}\right)(\mathrm{Ind})_{2}$ & $D M F$ & 0.13 & 0.00 & 0.02 & 0.19 & 0.08 & 0.23 & 0.11 & 0.17 & $25 b$ \\
\hline$m e r-\mathrm{Ru}$ (buim) $)_{3} \mathrm{Cl}_{3}$ & $D M F$ & -0.57 & -0.76 & -0.80 & -0.31 & -0.79 & -0.29 & -0.39 & -0.50 & $25 \mathrm{c}$ \\
\hline$m e r-\mathrm{Ru}(\mathrm{bim})_{3} \mathrm{Cl}_{3}$ & $D M F$ & -0.32 & -0.54 & -0.50 & -0.12 & -0.49 & -0.09 & -0.29 & -0.41 & $25 \mathrm{c}$ \\
\hline$m e r-\mathrm{RuCl}_{3}(\mathrm{Metrz})_{3}$ & $D M F$ & -0.11 & -0.23 & -0.22 & 0.02 & -0.22 & 0.03 & -0.06 & -0.18 & $25 \mathrm{c}$ \\
\hline$m e r-\mathrm{RuCl}_{3}(\mathrm{Mepz})_{3}$ & $D M F$ & 0.01 & -0.15 & -0.13 & 0.06 & -0.13 & 0.08 & -0.05 & -0.13 & $25 \mathrm{c}$ \\
\hline$m e r-\mathrm{RuCl}_{3}(\mathrm{pz})_{3}$ & $D M F$ & -0.01 & -0.09 & -0.06 & 0.17 & -0.06 & 0.18 & 0.09 & -0.10 & $25 \mathrm{c}$ \\
\hline$m e r-\mathrm{RuCl}_{3}(\mathrm{Ind})_{3}$ & $D M F$ & 0.08 & 0.02 & 0.03 & 0.28 & 0.03 & 0.29 & 0.22 & 0.10 & $25 \mathrm{c}$ \\
\hline mer,trans $-\mathrm{RuCl}_{3}\left(\mathrm{Me}_{2} \mathrm{~S}\right)(\mathrm{Ind})_{2}$ & DMSO & 0.15 & -0.21 & -0.20 & 0.26 & -0.20 & 0.27 & 0.22 & 0.18 & $25 b$ \\
\hline mer, trans $-\mathrm{RuCl}_{3}\left(\mathrm{Et}_{2} \mathrm{~S}\right)(\mathrm{Ind})_{2}$ & DMSO & 0.11 & -0.24 & -0.24 & 0.21 & -0.23 & 0.22 & 0.13 & 0.19 & $25 b$ \\
\hline$m e r-\mathrm{Ru}(\text { buim })_{3} \mathrm{Cl}_{3}$ & $D M S O$ & -0.50 & -1.03 & -1.04 & -0.33 & -1.03 & -0.32 & -0.37 & -0.46 & $25 \mathrm{c}$ \\
\hline$m e r-\mathrm{Ru}(\mathrm{bim})_{3} \mathrm{Cl}_{3}$ & DMSO & -0.29 & -0.81 & -0.76 & -0.12 & -0.75 & -0.10 & -0.27 & -0.38 & $25 \mathrm{c}$ \\
\hline$m e r-\mathrm{RuCl}_{3}(\mathrm{Metrz})_{3}$ & DMSO & -0.11 & -0.56 & -0.55 & -0.03 & -0.55 & -0.02 & -0.05 & -0.20 & $25 \mathrm{c}$ \\
\hline$m e r-\mathrm{RuCl}_{3}(\mathrm{Mepz})_{3}$ & $D M S O$ & 0.03 & -0.42 & -0.39 & 0.10 & -0.39 & 0.11 & -0.03 & -0.13 & $25 \mathrm{c}$ \\
\hline$m e r-\mathrm{RuCl}_{3}(\mathrm{pz})_{3}$ & DMSO & 0.03 & -0.32 & -0.30 & 0.17 & -0.29 & 0.18 & 0.10 & -0.11 & $25 \mathrm{c}$ \\
\hline$m e r-\mathrm{RuCl}_{3}(\mathrm{Ind})_{3}$ & DMSO & -3.33 & -0.07 & -0.08 & 0.41 & -0.07 & 0.42 & 0.24 & 0.09 & $25 \mathrm{c}$ \\
\hline$m e r-\mathrm{RuCl}_{3}(\mathrm{DMSO})_{2}\left(\mathrm{OH}_{2}\right)$ & water & 0.68 & 0.53 & 0.56 & 0.76 & 0.56 & 0.77 & 0.60 & 0.43 & $24 d$ \\
\hline$m e r-\mathrm{Ru}\left(\mathrm{NH}_{3}\right) \mathrm{Cl}_{3}(\mathrm{DMSO})_{2}$ & water & 0.69 & 0.50 & 0.52 & 0.69 & 0.52 & 0.69 & 0.62 & 0.36 & $24 d$ \\
\hline$m e r-\mathrm{RuCl}_{3}(\mathrm{DMSO})_{2}(\mathrm{Im})$ & water & 0.42 & 0.33 & 0.36 & 0.47 & 0.37 & 0.48 & 0.38 & 0.34 & $24 d$ \\
\hline$\left[\mathrm{Ru}(\mathrm{bpy}) \mathrm{Cl}_{4}\right]^{-}$ & $A C N$ & -0.19 & -0.84 & -0.90 & -0.07 & -0.89 & -0.06 & -0.07 & -0.08 & $24 \mathrm{i}$ \\
\hline$\left[\mathrm{Ru}(\mathrm{dcbpy}) \mathrm{Cl}_{4}\right]^{-}$ & $A C N$ & -0.01 & -0.56 & -0.55 & 0.09 & -0.54 & 0.09 & 0.10 & 0.08 & $24 \mathrm{i}$ \\
\hline trans $-\left[\mathrm{RuCl}_{4}(\mathrm{DMSO})(\mathrm{Im})\right]^{-}$ & $D M F$ & -0.16 & -0.38 & -0.38 & 0.01 & -0.37 & 0.03 & -0.10 & -0.22 & $25 \mathrm{a}$ \\
\hline
\end{tabular}




\begin{tabular}{|c|c|c|c|c|c|c|c|c|c|c|}
\hline trans $-\left[\mathrm{RuCl}_{4}(\mathrm{Im})_{2}\right]^{-}$ & $D M F$ & -0.58 & -0.85 & -0.90 & -0.31 & -0.88 & -0.29 & -0.40 & -0.72 & $25 \mathrm{c}$ \\
\hline trans $-\left[\mathrm{RuCl}_{4}(\operatorname{trz})_{2}\right]^{-}$ & $D M F$ & -0.36 & -1.33 & -1.34 & -0.15 & -1.33 & -0.13 & -0.16 & -0.48 & $25 \mathrm{c}$ \\
\hline trans $-\left[\mathrm{RuCl}_{4}(\mathrm{Ind})_{2}\right]^{-}$ & $D M F$ & -0.27 & -0.48 & -0.47 & 0.06 & -0.46 & 0.07 & -0.11 & -0.43 & $25 \mathrm{c}$ \\
\hline trans $-\left[\mathrm{RuCl}_{4}(\mathrm{Im})_{2}\right]^{-}$ & $D M S O$ & -0.59 & -1.46 & -1.46 & -0.35 & -1.45 & -0.33 & -0.35 & -0.74 & $25 \mathrm{c}$ \\
\hline trans $-\left[\mathrm{RuCl}_{4}(\operatorname{trz})_{2}\right]^{-}$ & DMSO & -0.37 & -1.22 & -1.20 & -0.18 & -1.20 & -0.18 & -0.12 & -0.44 & $25 \mathrm{c}$ \\
\hline trans $-\left[\mathrm{RuCl}_{4}(\mathrm{Ind})_{2}\right]^{-}$ & DMSO & -0.23 & -0.92 & -0.90 & 0.09 & -0.90 & 0.10 & -0.09 & -0.41 & $5 \mathrm{c}$ \\
\hline trans $-\left[\mathrm{RuCl}_{4}(\mathrm{Im})_{2}\right]^{-}$ & water & -0.47 & -0.92 & -0.83 & -0.20 & -0.83 & -0.19 & -0.30 & -0.15 & $24 f$ \\
\hline trans $-\left[\mathrm{RuCl}_{4}(\mathrm{tz})_{2}\right]^{-}$ & water & -0.28 & -0.62 & -0.63 & -0.09 & -0.63 & -0.08 & -0.12 & -0.01 & $24 \mathrm{~h}$ \\
\hline trans $-\left[\mathrm{RuCl}_{4}(\mathrm{Ind})_{2}\right]^{-}$ & water & -0.12 & -0.45 & -0.45 & 0.19 & -0.44 & 0.20 & 0.03 & 0.03 & $25 \mathrm{a}$ \\
\hline trans $-\left[\mathrm{RuCl}_{4}(\mathrm{DMSO})_{2}\right]^{-}$ & water & 0.18 & -0.15 & -0.11 & 0.31 & -0.11 & 0.32 & 0.28 & 0.47 & $24 \mathrm{c}$ \\
\hline trans $-\left[\mathrm{RuCl}_{4}(\mathrm{DMSO})\left(\mathrm{OH}_{2}\right)\right]^{-}$ & water & 0.06 & -0.19 & -0.17 & 0.21 & -0.17 & 0.21 & 0.25 & 0.35 & $24 \mathrm{c}$ \\
\hline trans $-\left[\mathrm{Ru}\left(\mathrm{NH}_{3}\right) \mathrm{Cl}_{4}(\mathrm{DMSO})\right]^{-}$ & water & -0.05 & -0.35 & -0.33 & 0.08 & -0.33 & 0.09 & 0.05 & 0.27 & $24 d$ \\
\hline trans $-\left[\mathrm{RuCl}_{4}(\mathrm{DMSO})(\mathrm{Im})\right]^{-}$ & water & -0.06 & -0.47 & -0.38 & 0.12 & -0.37 & 0.13 & 0.07 & 0.24 & $24 d$ \\
\hline trans $-\left[\mathrm{RuCl}_{4}(\mathrm{DMSO})(\mathrm{MeIm})\right]^{-}$ & water & -0.11 & -0.42 & -0.40 & 0.07 & -0.39 & 0.08 & 0.04 & 0.25 & $24 d$ \\
\hline trans $-\left[\mathrm{RuCl}_{4}(\mathrm{DMSO})(\mathrm{pz})\right]^{-}$ & water & 0.03 & -0.33 & -0.31 & 0.16 & -0.31 & 0.17 & 0.11 & 0.31 & $24 d$ \\
\hline trans $-\left[\mathrm{RuCl}_{4}(\mathrm{DMSO})(\mathrm{Ind})\right]^{-}$ & water & 0.06 & -0.28 & -0.27 & 0.18 & -0.27 & 0.19 & 0.16 & 0.33 & $24 d$ \\
\hline trans $-\left[\mathrm{RuCl}_{4}(\mathrm{DMSO})(\mathrm{py})\right]^{-}$ & water & -0.01 & -0.33 & -0.31 & 0.15 & -0.31 & 0.16 & 0.15 & 0.31 & $24 d$ \\
\hline trans $-\left[\mathrm{RuCl}_{4}(\mathrm{py})(\mathrm{TMSO})\right]^{-}$ & water & -0.03 & -0.37 & -0.37 & 0.15 & -0.36 & 0.16 & 0.12 & 0.30 & $24 d$ \\
\hline
\end{tabular}

a bim: benzimidazole, bpy: 2,2'-bipyridine, 4,4'-bpy: 4,4'-bipyridine, buim: 1-butylimidazole, dcbpy: 4,4'-dicarboxylic acid-2,2'bipyridine, $\mathrm{Et}_{2} \mathrm{~S}$ : diethyl sulfide, $\mathrm{Me}_{2} \mathrm{~S}$ : dimethyl sulfide, DMSO: dimethyl sulfoxide, en: ethylenediamine, Im: imidazole, Ind: indazole, isn: isonicotinamid, Meim: 4-methylimidazole, Mepz: 4-methylpyrazole, Metrz: 1-methyl-1,2,4-triazole, py: pyridine, pz: pyrazole, trz: 1,2,4-triazole, Metrz: 1-methyl-1,2,4-triazole, TMSO: tetramethylene sulfoxide, tz: thiazole. 
Figure S-1. Calculated vs experimental SRPs. (a) Protocol III, PCM/UAKS, (b) Protocol V, CPCM/UAKS, (c) Protocol VI, CPCM/Bondi. The data are sorted by solvent (color of symbol) and charge (shape of symbol). Aqueous SRPs of the medicinally relevant complexes with the lead structure, trans-[ $\left.\mathrm{RuCl}_{4}(\mathrm{~L})\left(\mathrm{L}^{\prime}\right)\right]^{-}$, are displayed as filled symbols.
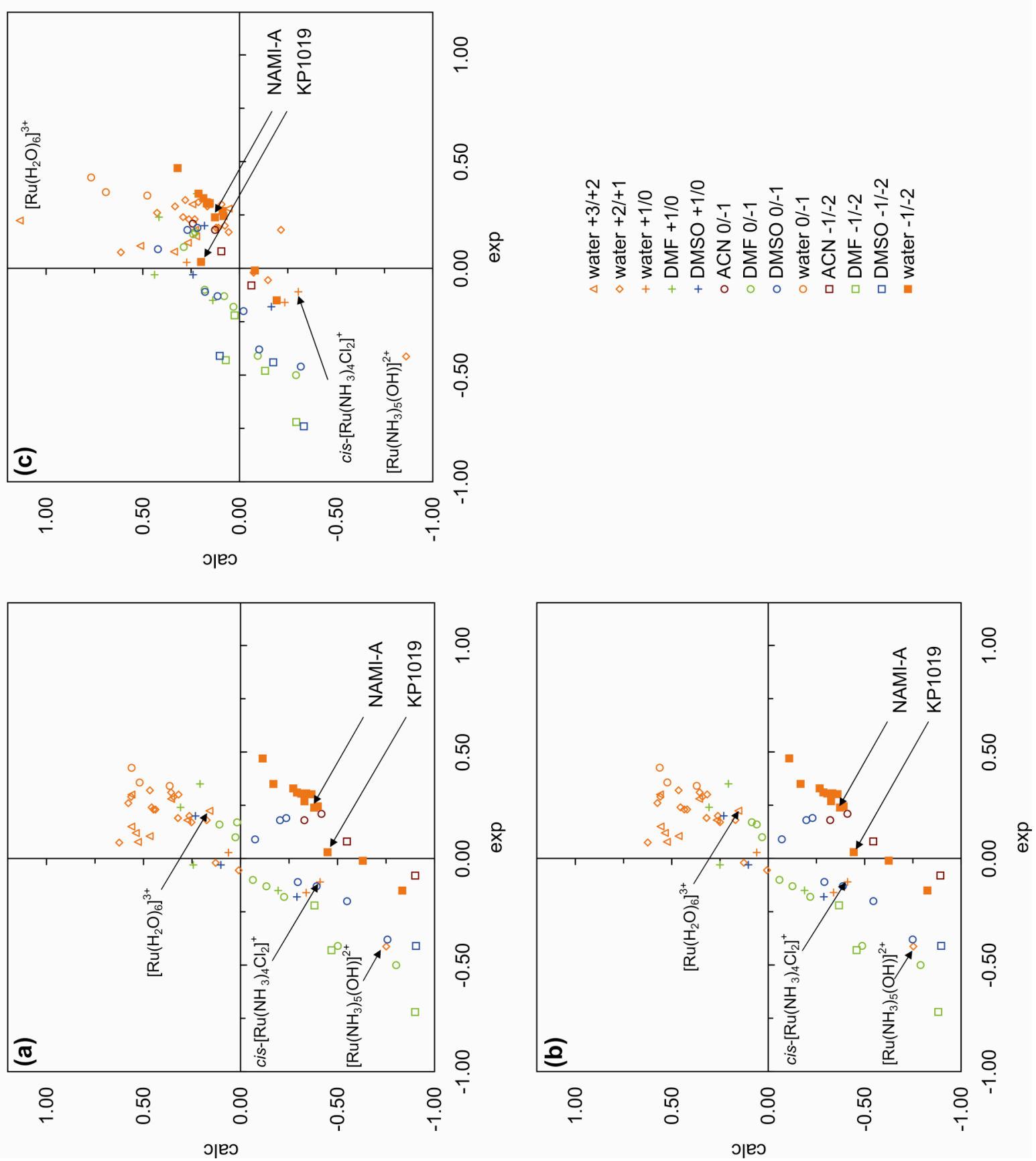